\title{
Observations on the Tube Method of Measuring Growth Rate in Neurospora crassa
}

\author{
By O. J. GILLIE* \\ Institute of Animal Genetics, West Mains Road, Edinburgh 9
}

(Accepted for publication 30 August 1967)

SUMMARY

The relationship between various parameters of growth of Neurospora crassa on solid media in tubes is described. These are $(a)$ the relationship between culture weight and linear growth rate on various media; $(b)$ the relationship between 'lag' in growth and inoculum size on various media. This latter relationship appears to obey a log law, enabling an inoculum doublingtime to be calculated. Adaptation of the arginine auxotroph arg-I (46004) to diminution of arginine in the medium first occurred by decrease in mycelial weight and then by decrease in linear growth rate. The inoculum doublingtime did not appear to vary systematically with arginine concentration.

\section{INTRODUCTION}

The tube method of measuring growth of fungi on lengths of solid medium has been widely used in auxanographic studies and bioassays; a comprehensive study of many aspects of this method was published by Ryan, Beadle \& Tatum (I943). However, as pointed out by Pirt (1967), the laws which govern the growth of bacterial and fungal colonies remain to be elucidated. Studies of this type of growth are of importance because, not only in the laboratory but also in nature, micro-organisms frequently grow in a colonial or spreading fashion on solid media; furthermore, growth of this type is of interest as it is the simplest model system which approaches the kind of growth which must occur in the tissues of higher organisms.

The purpose of the present work was to search for relationships between the simplest observable variables concerned with growth of fungi on lengths of solid media in tubes: the independent variables were inoculum size and medium composition, and the dependent variables were linear growth rate, lag in growth and mass of growth. The relationship between mass of growth and linear growth rate (outward growth of the colony) was studied for various strains of Neurospora crassa grown on various nutrient agar media in tubes. Measurements of lag in growth on tubes were found to be dependent on the inoculum size, and a new parameter called the inoculum doublingtime has been derived from this relationship. The comparison of the way in which these parameters vary together proved helpful in understanding the nature of growth of fungal cultures on the surface of solid media and the way in which growth adapts to dilution of an essential nutrient.

\footnotetext{
* Present address: National Institute for Medical Research, Mill Hill, London, N.W. 7.
} 


\section{METHODS}

Growth tubes of the type described by Brown \& Gillie (1963) were used (Fig. I). The essential features of these tubes were as follows: the ends were straight enabling a wire to be introduced and used to take a sample of material from any part of the tube, the agar medium ( $10 \mathrm{ml}$.) was introduced into the tube through a central chimney (which may also be used for taking samples of conidia in other kinds of experiments) and was retained in the tube by an indented ridge in the glass $4 \mathrm{~cm}$. from the end of the tube. The distance between the indented ridges in which the solid medium lies was $29-30 \mathrm{~cm}$. Soda or Perspex glass may be used with an inside diameter of $12 \mathrm{~mm}$. for the body of the tube and $8 \mathrm{~mm}$. for the chimney. To maintain the tubes in an upright position they have two small glass feet attached at the base half way along the tube. Not only are these tubes more versatile than the classical type, they are also more convenient to handle. They have been made for us by a glassblower at the University at an estimated cost, including overheads of 6 shillings each.

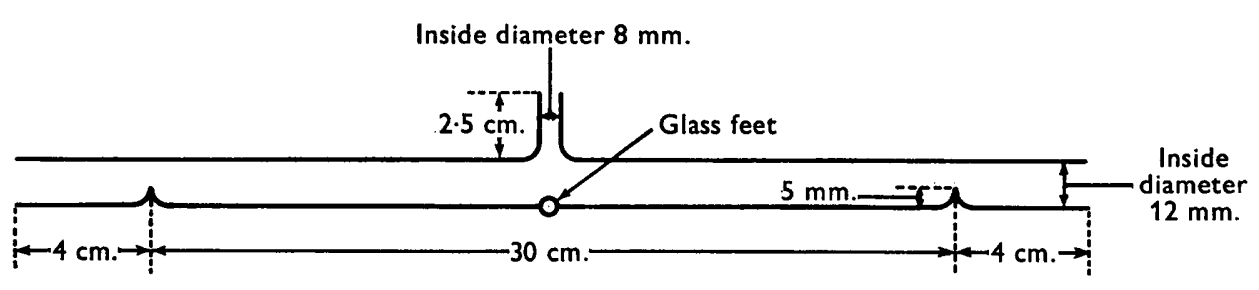

Fig. I. Growth tube.

The ends of the tubes are plugged with cottonwool and they are dry sterilized before molten agar is pipetted into them. Cleaning of the tubes is greatly facilitated if they are soaked for about 2 days in a mixture of detergent and bleach, after which the agar can usually be easily dislodged by running hot tap water through the tube. A stout wire (with a flattened and bent end) was used to remove sections of the agar and adhering growth of Neurospora from the tube for experimental purposes. The mass of mycelium in an individual section or a number of pooled sections could be determined by weighing after extraction of the agar. To extract the agar $5 \%$ trichloroacetic acid was added to the sections ( $\mathrm{I} \mathrm{ml}$. to every $\mathrm{ml}$. of agar medium) and the mixture was heated for ro min. in a boiling water bath. The mycelium was then removed by filtration through paper on a Buchner funnel and could be freeze-dried and weighed. Weighings of less than $2 \mathrm{mg}$. were made with a Cahn electrobalance. Studies of the extinction of the extraction liquor at 260 and $280 \mathrm{~m} \mu$ after removal of trichloroacetic acid with ether showed that between 5 and $10 \%$ by weight of ribonucleotides were removed by this treatment, but this did not vary sufficiently with the age of the section to influence the results.

Inoculations of the tubes were made by using a calibrated welded wire loop which delivered a mean volume of $\mathrm{I} \cdot 8 \mu \mathrm{l}$. (with a standard error of $\pm 0.6 \mu \mathrm{l}$.). The inoculum was suspended in distilled water, filtered through cottonwool, counted in a haemocytometer and then appropriately diluted in distilled water. The point of inoculation was marked, and daily markings of growth were made subsequently.

Standard Vogel (Vogel, 1956 medium $\mathrm{N}$ with $2 \%(\mathrm{w} / \mathrm{v})$ sucrose and $2 \%(\mathrm{w} / \mathrm{v})$ 
Difco Bactoagar were used unless otherwise stated. Various strains were used, including our strains of the Emerson wild type EMA and St Lawrence wild type STA. Strains $\arg$-I $A$ (46004) and $\operatorname{arg-IOa}$ (в 362) had mixed Emerson-St Lawrence backgrounds. Neither of these arginine auxotrophs was at all leaky on minimal medium. All experiments were done at $25^{\circ}$. Media were supplemented as indicated with arginine $\mathrm{HCl}$ obtained from British Drug Houses Ltd.

\section{RESULTS}

\section{Measurements of the mass of mycelium growing in tubes}

The mass of mycelium growing in I-cm. long sections pooled from Io tubes was determined at measured distances from the growing front. Figure $2 a$ and $2 b$ shows

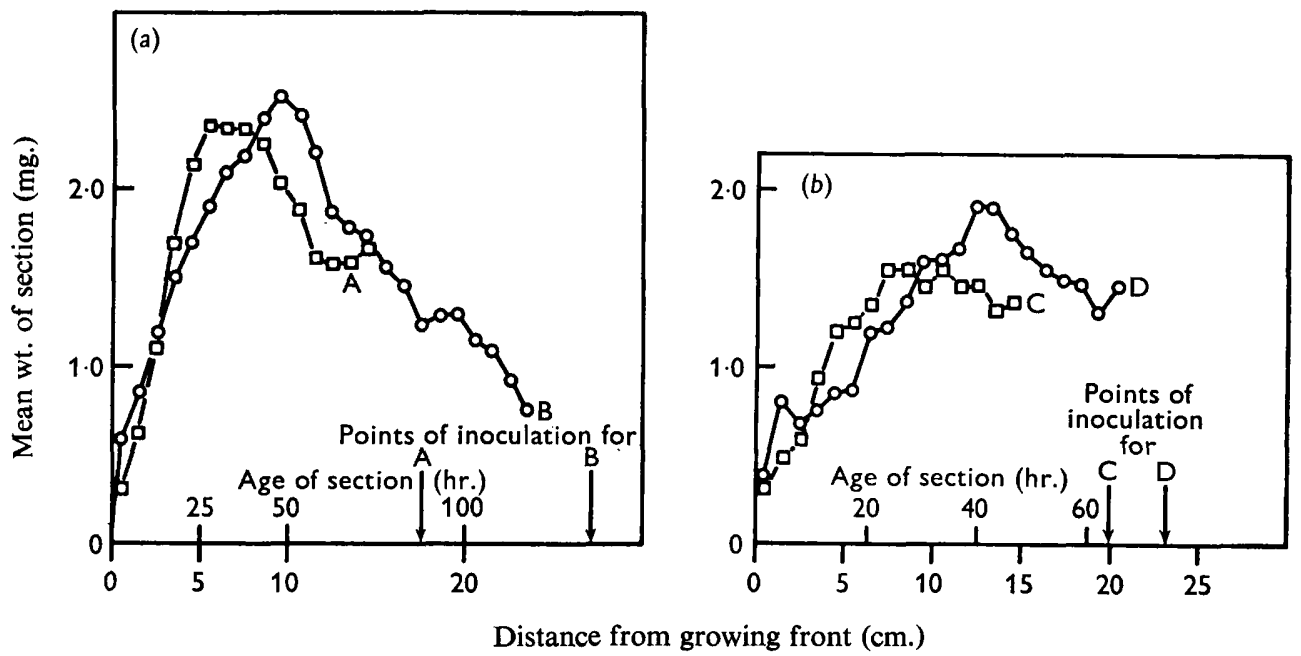

Fig. 2. (a) Yield during linear growth in tubes of Neurospora crassa wild-type strain EMA. Sections were cut at $I \mathrm{~cm}$. intervals, starting at the growing front and sections from the same position in each of 10 tubes were pooled and weighed. The mean weight of the section has been plotted at its median distance from the growing front. The mean age of the section is also given as the alternative abscissa. Two experiments (A and B) are shown in which growth occurred over different lengths of tube. These have been superimposed at the growing front to facilitate comparison. The points of inoculation are indicated on the abscissa. (b) Yield during growth in tubes of Neurospora crassa wild-type strain STA. Experimental procedure as described in (a). Two separate experiments, $C$ and $D$, were made.

that with the Emerson wild-type strain, and less clearly with the St Lawrence wildtype strain, the mycelium increased more or less linearly in mass for 5-10 cm. behind the growing front and thereafter a decrease in mycelial mass was found. The peak of mycelial mass occurred at more or less the same distance from the growing front, regardless of the distance from the point of inoculation. A similar situation was found with all the strains tested, although the peak came a little later and declined less sharply with strain STA (Fig. $2 b$ ).

Growth of this kind may be visualized as a wave of mass proceeding along the tube and in a sense eventually passing off the end of the tube. This view is supported by measurements of the total extractable mycelial mass in a tube, which was found to 
increase until the mycelial front reached the end of the tube, and then decreased. This type of wave-like growth of the perimeter of a 'colony' has obvious similarities to the kind of growth which may in nature lead to the production of 'fairy rings' by certain types of fungi.

The mycelial mass produced varied with dilution of a growth-limiting nutrient. Figure 3 shows growth curves of mycelial mass for the arginine auxotroph, arg-I (46004) grown on different concentrations of arginine. The mycelial mass recovered declined with dilution of arginine in the medium, as might be expected, although at very high concentrations of arginine ( $5 \mathrm{~g}$. arginine $\mathrm{HCl} / \mathrm{l}$.) there was a more gradual increase in mycelial mass along the tube, showing some sort of adaptation. The relation-

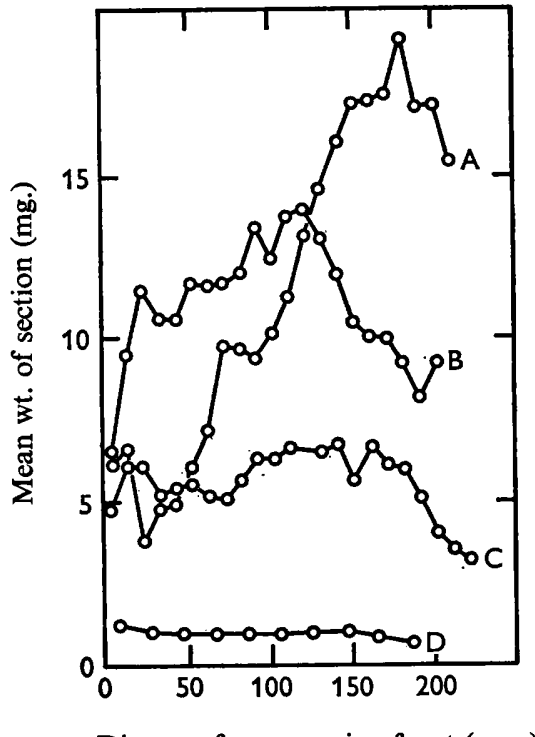

Distance from growing front (mm.)

Fig. 3

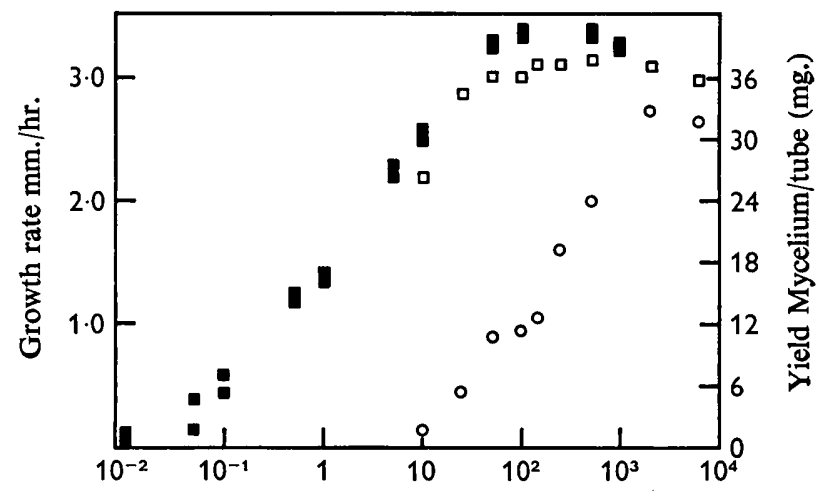

Arginine $\mathrm{HCl}$ (mg./l.)

Fig. 4

Fig. 3. Yield during growth in tubes of Neurospora crassa strain arg-I grown on various arginine concentrations. Experimental procedure was as described in the legend of Fig. I $a$. The arginine concentrations used were: $\mathrm{A}, 5 \times 10^{3} \mathrm{mg} . / 1$.; $\mathrm{B}, 5 \times 10^{2} \mathrm{mg} . / 1$. $\mathrm{C}, \mathrm{I} \cdot 5 \times 10^{2} \mathrm{mg} . / 1$; D, $10 \mathrm{mg} . / 1$.

Fig. 4. The relationship between linear growth rate and yield of Neurospora crassa strain arg-I and arginine concentration in the medium. $O$, measurements of yield of mycelium per tube, averaged from to tubes at each arginine concentration. $\square$, measurements of linear growth rate in individual tubes. $\square$, average measurements of linear growth rate (from 10 tubes) made from the same tubes as the measurements of yield were made.

ship between mycelial mass measured in this way and linear growth rate is shown in Fig. 4. This shows that as the concentration of arginine in the medium decreased there was a 5-fold decrease in yield of mycelial mass before the linear growth rate started to decrease. This type of relationship between yield of mycelial mass and linear growth rate was found with all of several strains grown on various media. Figure 5 shows linear growth rate plotted directly against yield for all the strains and conditions tested, showing that, whether salts, sugar or arginine (in the case of arginine auxotrophs) were the growth-limiting nutrient, the yield decreased to about Io mg. 
mycelium/tube before the linear growth rate started to decrease significantly. It was not found practical to measure the very low yields obtained at low growth rates and so the relationship between the two parameters was not explored below about I mg. mycelium/tube.

\section{The relationship between inoculum size and lag in growth}

In a previous paper (Gillie, 1968) the relationship between inoculum size and growth in exponential cultures of micro-organisms was considered and it was shown

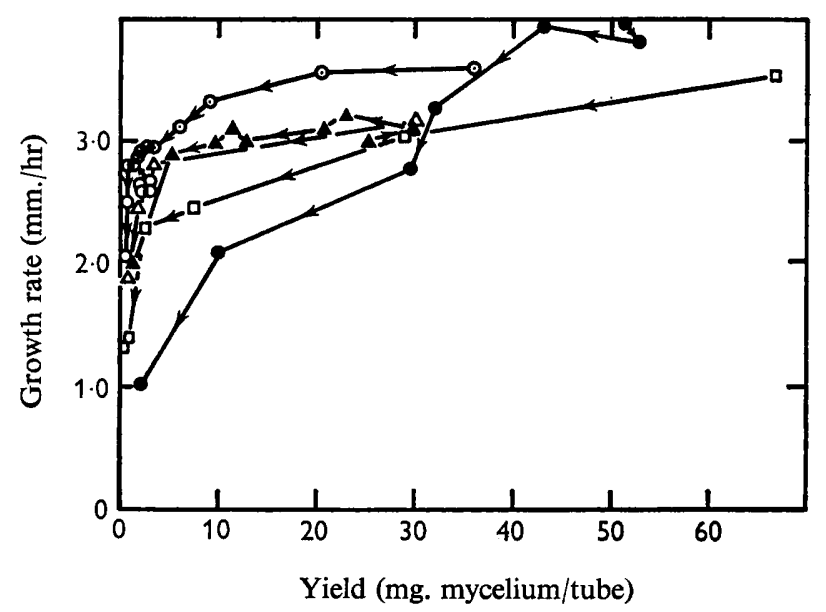

Fig. 5. Linear growth rate is plotted against yield of mycelium per tube for several strains of Neurospora crassa growing under widely different conditions. Each experiment, represented by one type of symbol on the graph, was made with one strain growing on a medium in which one constituent was progressively diluted. The direction of progressive dilution is represented on the graph by the arrows on the lines connecting points. The strains and media used were as follows: 9 , strain $\arg -10 \mathrm{on} 500 \mathrm{mg}$. arginine/l. and these concentrations of Vogel salt solution: $2 \cdot 0,1 \cdot 5,1 \cdot 0,0.5,0.1,0.01 \mathrm{ml} . / 100 \mathrm{ml}$. medium. $O$, strain arg-IO on medium with no sucrose and these concentrations of arginine $10,5,1 \cdot 0,0.5,0 \cdot 1$, $0.05,0.01,0.005 \mathrm{~g} . / 1$. $\odot$, strain arg-Iogrown on $500 \mathrm{mg}$. arginine/l. and these concentrations of sucrose: $20,5, \mathrm{I}, 0.5,0 . \mathrm{I}, 0.05,0.0 \mathrm{I}, 0 \mathrm{~g} . / \mathrm{l} . \triangle$, strain $\arg$-I0 on these concentrations of arginine: $0.5,0.04,0.01,0.005 \mathrm{~g} . / 1$. $\Delta$, strain $\arg$-I on these concentrations of arginine: $5 \cdot 0,2 \cdot 0$, $0.5,0.25,0.175,0.05,0.025,0.01 \mathrm{~g} . / 1$. $\square$, wild-type strain STA on Roberts medium (Roberts $e t$ al. 1955) with these concentrations of sucrose: $20,10,5.0,2 \cdot 0,0.5,0.2,0.1 \mathrm{~g} . / 1$. Roberts medium contains only inorganic salts with no citrate which was present in the Vogel salt solution and might have acted as a subsidiary carbon source.

that when the inoculum size was varied in a series of cultures and the weight of each culture after growth considered as a constant value (in practice by interpolating between experimental values), then from the exponential growth equation

$$
\log _{e} M-\log _{e} M_{0}=\mu t
$$

(where $M_{0}$ is mass of inoculum and $M$ is the mass of the culture at time $t$ ), it can be seen that if $M$ is treated as constant, $-\log _{e} M_{0}$ is proportional to $t$. A relationship of this type was found to hold between 'lag' (time after inoculation when growth first becomes measurable) and inoculum size for linear growth of Neurospora in tubes. The experimental investigation of this relationship is described below.

Tubes of minimal medium supplemented with different quantities of L-arginine 
were inoculated with from I to $10^{5}$ conidia of the $\arg$-I or arg-10 mutant strain of Neurospora crassa. The inoculum was diluted in 5 -fold steps, and each inoculation at a particular conidial concentration (there were from 5 to 7 such conidial concentrations for each arginine concentration) and a particular arginine concentration was made in duplicate. It was found that at a particular arginine concentration the linear growth rate $(\mathrm{mm}$./hr) did not vary with inoculum size (see Fig. 6). However, as the inoculum became more dilute there was a progressive delay in the initiation of linear growth (see Fig. 6). Extrapolation of the line describing this growth to zero on the length of growth axis served to define the parameter which has been called the lag and which is measured

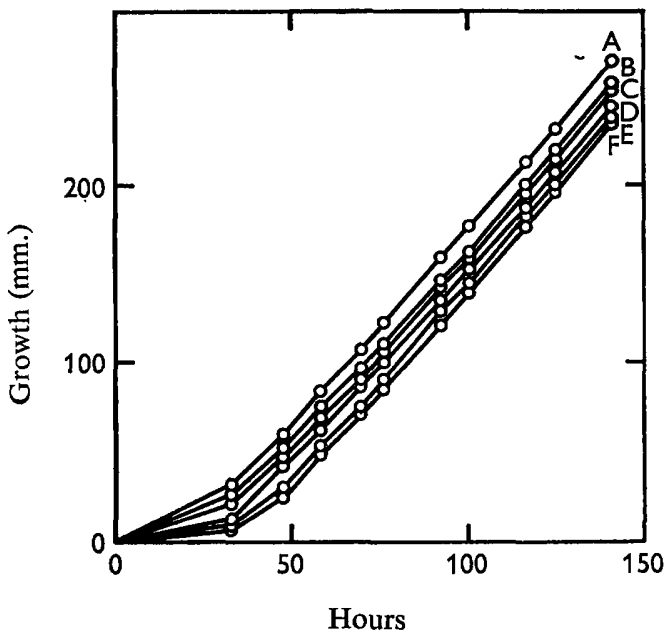

Fig. 6

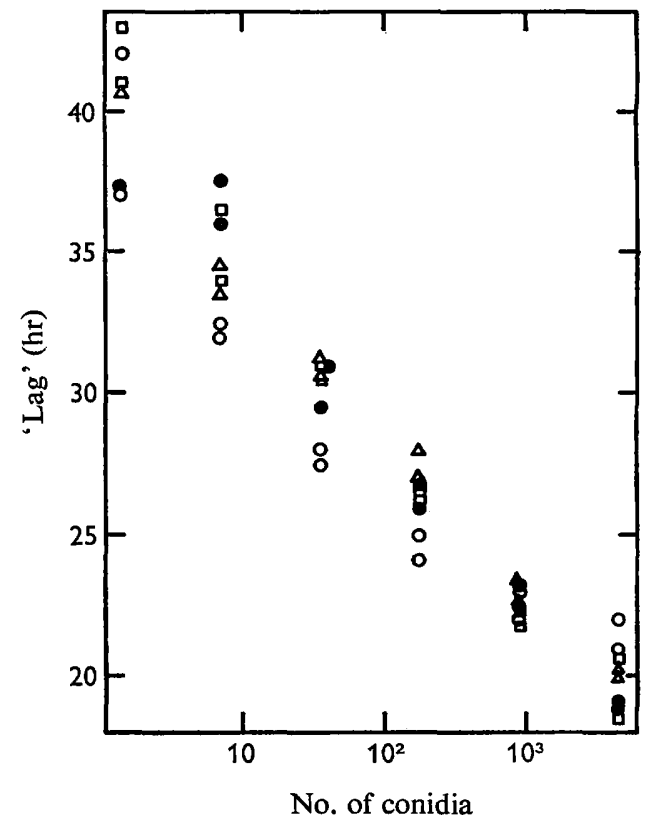

Fig. 7

Fig. 6. Growth of Neurospora crassa strain arg-1 in growth tubes at various inoculum dilutions. Results are shown from only one of two duplicate tubes at each inoculum dilution on $5 \mathrm{mg}$. arginine/l. Calculated inocula (conidia/tube) were: $\mathrm{A}, 4.4 \times 10^{3} ; \mathrm{B}, 8.8 \times 10^{2} ; \mathrm{C}, 176$; D, 35; E, 7; F, I. Lags estimated from these data are plotted against inoculum size in Fig. 6 .

Fig. 7. Lag is plotted against log inoculum size for Neurospora crassa strain arg-I grown with various concentrations of arginine in the medium. indicate data from medium containing $5 \mathrm{~g}$. arginine $/ 1 . \triangle, 2 \mathrm{~g}$. arginine $/ 1 . \square, \mathrm{I} 00 \mathrm{mg}$. arginine/l. $O, 5 \mathrm{mg}$. arginine/l. These data appear as series $\mathrm{D}$ in Table $\mathrm{I}$.

in hours. The lag then is the time (in hours) taken for growth to be initiated after inoculation, assuming that all growth occurs at the same linear rate. Since growth does occur at the same linear rate, once established, the lag may be readily determined by extrapolation from a graph by using a ruler.

Regressions were calculated of lag against log inoculum size and it was found that there was a linear relationship for arginine concentrations above $3 \mathrm{mg}$. $/ 1$. These results are shown in Table I. The relationship showed a significant non-linearity $(P<0.0 \mathrm{I})$ in five cases. but this was found to be almost wholly due to the inclusion of inocula 
with a calculated number of Io conidia or less per tube. When inocula of Io conidia or less were excluded from the calculations then only one out of fifteen regressions showed significant non-linearity $(P=0.001$ to 0.01$)$; however, this is not significant when all the experiments are considered together. It is not perhaps surprising that the lag showed greater variability for calculated inocula of Io conidia or less, since in small samples the percentage variation in actual numbers per tube and the variation in time of germination of individual spores will be greater.

Table I. Neurospora crassa strains arg-I and arg-Io (arginine-dependent). Calculated regressions in lag in linear growth against log number of conidia in inoculum

\begin{abstract}
Results of the type illustrated in Fig. 7 were collected for strains arg-I and arg-Io grown on various concentrations of arginine in growth tubes, and were used to calculate the slopes of lag in growth (in $\mathrm{hr}$ ) plotted against log inoculum dilution. The slope is measured by the regression constant $b$ which is defined as lag in $\mathrm{hr} / \mathrm{ro}^{-1}$ dilution of inoculum. The experiments were performed in several series (A to $F$ ); each series of experiments was done at the same time with the same inoculum dilutions. Inocula with a calculated size of Io conidia or less have been omitted in calculating these regressions (see text).
\end{abstract}

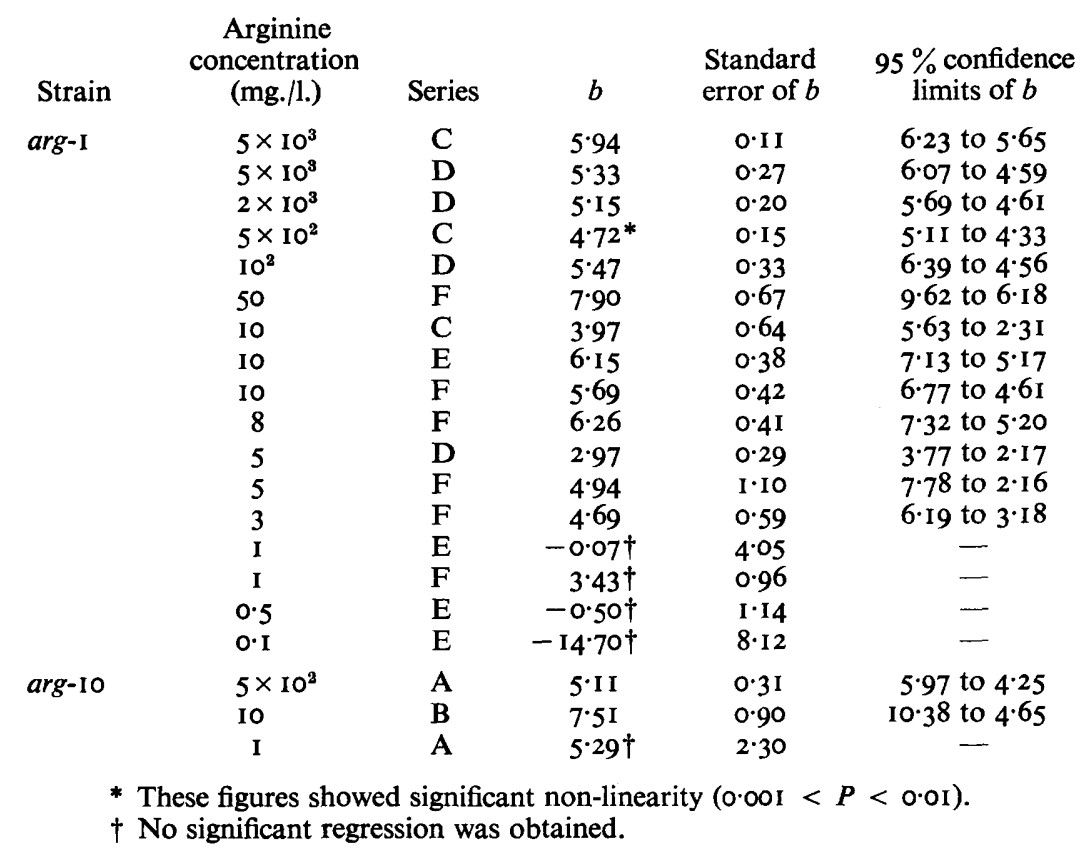

The linear relationship between log of inoculum size and lag was used to calculate the regression constant $b$ (Table $\mathrm{I}$ ) which is expressed as lag in hours per $10^{-1}$ dilution of inoculum. An unweighted mean estimate of $b$ for $\arg$-I grown on 3 to $5 \times 10^{3} \mathrm{mg}$. arginine/l. was calculated as $5.32 \mathrm{hr} / \mathrm{IO}^{-1}$ dilution of inoculum $(95 \%$ confidence limits 5.98 to 4.66 ), and the mean for arg-Io grown on IO and $5 \times \mathrm{IO}^{2} \mathrm{mg}$. arginine $/ 1$. was found to be $5 \cdot 37(95 \%$ confidence limits $6 \cdot 25$ to $4 \cdot 49)$. These mean estimates were compared and found to be homogeneous $(0.7<P<0.8)$ and have been combined to give a weighted mean estimate of $5.35 \mathrm{hr} / \mathrm{IO}^{-1}$ dilution of inoculum ( $95 \%$ confidence limits $5 \cdot 78$ to $4 \cdot 9 \mathrm{I}$ ). This weighted mean estimate was used to calculate a doubling time 
of $\mathrm{I} \cdot 6 \mathrm{I} \mathrm{hr}(95 \%$ confidence limits $\mathrm{I} \cdot 48$ to $\mathrm{I} \cdot 74 \mathrm{hr})$. This may be thought of as the time it takes for an inoculum of $n$ conidia to grow to the effective size of an inoculum of $2 n$ conidia, where $n$ in practice lies between 10 and $10^{5}$. The method of calculation of doubling time from inoculum dilution data is discussed by Gillie (1967).

Considerable heterogeneity was found amongst the different estimates of $b$ (see Table 2); this was least amongst $b$ values estimated at the same arginine dilution. This suggests that there was an additional source of variability operating between experiments, but the variability found within experiments at particular arginine dilutions was much less than the variability found when the experiments were grouped in any other way. This heterogeneity makes the interpretation of the relationship between $b$ values (or inoculum doubling time) and arginine concentration difficult; however, it seems that there is no clear systematic relationship detectable between them over a range of 3 to $5 \times 10^{3} \mathrm{mg}$. arginine $/ 1$. and it is possible that the observed variation in $b$ values is mostly attributable to experimental error. No significant regression was obtained for $\log$ of inoculum size against lag for arg-I or arg-Io growing on concentrations of arginine of I mg./l. or less, although sustained growth was obtained on these low arginine concentrations.

Table 2. The homogeneity of $b$ values

\begin{tabular}{|c|c|c|c|c|}
\hline & & $\begin{array}{l}\text { Degrees } \\
\text { of free- } \\
\text { dom }\end{array}$ & $\chi^{2}$ & Probability \\
\hline Division A & $\begin{array}{l}\text { Between series } \\
\text { Within series } \\
\text { Total }\end{array}$ & $\begin{array}{r}4 \\
8 \\
12\end{array}$ & $\begin{array}{r}71 \cdot 80 \\
60 \cdot 43 \\
132 \cdot 23\end{array}$ & $\begin{array}{l}<0.001 \\
<0.001 \\
<0.001\end{array}$ \\
\hline Division B & $\begin{array}{l}\text { Between dilutions } \\
\text { Within dilutions } \\
\text { Total }\end{array}$ & $\begin{array}{r}8 \\
4 \\
12\end{array}$ & $\begin{array}{r}116 \cdot 29 \\
15 \cdot 94 \\
132 \cdot 23\end{array}$ & $\begin{array}{c}<0.001 \\
0.001 \text { to } 0.01 \\
<0.001\end{array}$ \\
\hline
\end{tabular}

\section{DISCUSSION}

The results described show that the strains of Neurospora crassa investigated adapted to decreasing concentrations of an essential nutrient in solid medium by a decrease in the mass of the culture followed by a decrease in linear growth rate. Both these parameters decreased roughly in proportion to the log of the arginine concentration, but the decrease in linear growth rate did not begin until a more than 5 -fold decrease in mycelial yield per tube had occurred. The log relationship between yield and arginine concentration cannot continue to be proportional in the same way below $10 \mathrm{mg}$. arginine/l. since growth occurs even on $10^{-2} \mathrm{mg}$. arginine/l. and at a measurable rate. It is interesting that it is at about this point (10-50 mg. arginine/1.), at which the rate of decrease in yield with decrease in arginine concentration must level off, that the decrease in linear growth rate begins. This suggests, in conjunction with the results of other experiments in which salts and sucrose as well as arginine were used as growth limiting nutrients, that when the yield of mycelium per tube reaches about 5-10 mg. the linear growth rate starts to decrease. This relationship between yield and growth rate indicates perhaps that there is a control mechanism to maintain the mycelium as near as possible to an optimal density suitable to the habit of growth of the organism. 
Growth on solid media has some superficial resemblances to growth in continuous culture in a chemostat, in that the mycelium as it grows is constantly being presented with new food, and also the yield of the organism may vary over a wide range as a result of varying the concentration of nutrients. In a chemostat, however, the yield is directly proportional to the concentration of the limiting nutrient (Monod, 1942), not proportional to the log of the limiting nutrient, as in linear growth in tubes. Linear growth rate also seems to be proportional to the log of the growth limiting nutrient (arginine). A similar non-linear relationship was found by Pirt (1967) between the initial colony radial growth rates of bacteria and the glucose concentration in the medium. Pirt pointed out that the initial concentration of the growth-limiting nutrient cannot be the concentration to which the bacteria are in fact exposed during most of their growth, because a concentration gradient must develop in the nutrient agar. These considerations probably also apply to the linear growth of Neurospora, as here studied, although the linear growth rate is much greater and mycelial 'roots' penetrate right through the agar to obtain nutrients more actively.

Models of spherical growth of fungi in liquid culture (discussed by Gillie, 1968), which assume a constant rate of increase of the radius of the sphere, are based on the linear rate of increase of the radius of fungal colonies on solid media, and tend to assume that the density of the organism is uniform throughout the 'sphere of growth' considered. However, the results described here show that an increase in yield takes place behind the growing front to a maximum value, after which a decrease in yield occurs. Future models of spherical growth of fungi may find it necessary to consider complications of this kind.

The inoculum doubling-time as calculated above is remarkably close to the inoculum doubling-time determined (by using the same reasoning) for growth in unstirred liquid cultures (Gillie, I968) of $\mathrm{r} \cdot 84 \mathrm{hr}$ for Neurospora strain STA (95\% confidence limits $\mathrm{I} \cdot 74$ to $\mathrm{I} \cdot 94 \mathrm{hr}$ ) and in reasonable agreement with doubling times for strain STA, grown in shaken logarithmic cultures, of $2 \cdot 3 \mathrm{hr}(95 \%$ confidence limits $\mathrm{I} \cdot 7-2 \cdot 9 \mathrm{hr})$ (C. F. Curtis, personal communication).

In one mutant strain of Neurospora crassa arg-I I (30820) which requires either 30\% $(\mathrm{v} / \mathrm{v})$ carbon dioxide or arginine + purine + pyrimidine for normal growth (Broadbent \& Charles, 1965), an inoculum doubling-time of $15 \mathrm{hr}$ for 'leaky growth' on minimal medium was observed as compared with an inoculum doubling-time of $3 \mathrm{hr}$ on complete medium. Once growth was established after the lag on these media the density of growth and the linear growth rates were in the same range. The slow rate of inoculum doubling in this strain arg-I I (30820) on minimal medium may be due to an initial dependence on small quantities of atmospheric carbon dioxide for growth, whereas later growth is able to rely on carbon dioxide generated by the organism itself.

The EMA wild-type strain of Neurospora crassa had a consistently lower linear growth rate $(\mathrm{I} .9 \mathrm{~mm} . / \mathrm{hr})$ than the STA wild-type strain $(3.2 \mathrm{~mm} . / \mathrm{hr})$ and yet the EMA wild-type strain gave consistently the highest yield of mycelium per tube $(42 \cdot 6 \mathrm{mg}$. as opposed to $32.4 \mathrm{mg}$. for strain STA). These results show that it cannot be assumed that a low linear growth rate indicates a lower yield when comparisons are made between strains.

I gratefully acknowledge the excellent technical assistance of Miss C. Stake and Mrs K. Henderson and the invaluable statistical advice and assistance of Miss J. Skegg. 


\section{REFERENCES}

Broadbent, J. A. \& Charles, H. P. (1965). Some carbon-dioxide requiring mutants of Neurospora crassa. J. gen. Microbiol. 39, 63.

Brown, I. R. \& GILLIE, O. J. (1963). A convenient design of growth tube. Neurospora Newsletter 4 , 19. GILIIE, O. J. (1968). Growth of Neurospora crassa in unstirred liquid cultures. J. gen. Microbiol. 5I, 179.

PIRT, S. J. (1967). A kinetic study of the mode of growth of surface colonies of bacteria and fungi. J. gen. Microbiol. 47, 181.

Monod, J. (1942). Recherche sur la Croissance des Cultures Bactériennes. Paris: Hermann et Cie.

Roberts, R. B., Abelson, P. H., Cowie, D. B., Bolton, E. T. \& Britten, R. J. (1955). In Studies of Biosynthesis, Publs. Carneg. Instn. No. 607.

Ryan, F. J., BEADLE, G. W. \& TATUM, E. L. (I943). The tube method of measuring the growth rate of Neurospora. Am. J. Bot. 30, 784 .

VoGEL, H. J. (1956). A convenient growth medium for Neurospora (medium N). Microb. Genet. Bull. 13, 42. 